Pacific Journal of Mathematics

THE NUMER OF MULTINOMIAL COEFFICIENTS DIVISIBLE 


\section{THE NUMBER OF MULTINOMIAL COEFFICIENTS DIVISIBLE BY A FIXED POWER OF A PRIME}

F. T. Howard

In this paper some results of $\mathrm{L}$. Carlitz and the writer concerning the number of binomial coefficients divisible by $p^{j}$ but not by $p^{j+1}$ are generalized to multinomial coefficients. In particular $\theta_{j}(k ; n)$ is defined to be the number of multinomial coefficients $n ! / n_{1} !, \cdots, n_{k}$ ! divisible by exactly $p^{j}$, and formulas are found for $\theta_{j}(k ; n)$ for certain values of $j$ and $n$. Also the generating function technique used by Carlitz for binomial coefficients is generalized to multinomial coefficients.

1. Introduction. Let $p$ be a fixed prime and let $n$ and $j$ be nonnegative integers. L. Carlitz [2], [3] has defined $\theta_{j}(n)$ as the number of binomial coefficients

$$
\left(\begin{array}{l}
n \\
r
\end{array}\right) \quad(r=0,1, \cdots, n)
$$

divisible by exactly $p^{j}$ and he has found formulas for $\theta_{3}(n)$ for certain values of $j$ and $n$. In particular, if we write

$$
n=a_{0}+a_{1} p+\cdots+a_{s} p^{s} \quad\left(0 \leqq a_{i}<p\right)
$$

then

$$
\begin{aligned}
& \theta_{0}(n)=\left(a_{0}+1\right)\left(a_{1}+1\right) \cdots\left(a_{s}+1\right) \\
& \theta_{1}(n)=\sum_{i=0}^{s-1}\left(a_{0}+1\right) \cdots\left(a_{i-1}+1\right)\left(p-\alpha_{i}-1\right) a_{i+1}\left(a_{i+2}+1\right) \cdots\left(a_{s}+1\right) .
\end{aligned}
$$

The writer [5], [6] has also considered the problem of evaluating $\theta_{j}(n)$.

The purpose of this paper is to consider the analogous problem for multinomial coefficients and to generalize some of the formulas developed by Carlitz and the writer. Thus we define $\theta_{j}(k ; n)$ as the number of multinomial coefficients

$$
\left(n_{1}, \cdots, n_{k}\right)=\frac{n !}{n_{1} ! \cdots n_{k} !}\left(n_{1}+\cdots+n_{k}=n\right)
$$

divisible by exactly $p^{j}$. In this definition the order of the terms $n_{1}, \cdots, n_{k}$ is important. We are distinguishing, for example, between $(1,2,3)$ and $(2,1,3)$. Clearly $\theta_{j}(2 ; n)=\theta_{j}(n)$.

In this paper we find formulas for $\theta_{0}(k ; n), \theta_{1}(k ; n)$, and $\theta_{2}(k ; n)$. We also show how the generating function method used by Carlitz 
can be generalized to multinomial coefficients, and we evaluate $\theta_{j}(k ; n)$ for special values of $j$ and $n$.

Throughout this paper we assume $p$ is a fixed prime number and $k$ is a fixed positive integer, $k>1$.

2. Preliminaries, Let $E\left(n_{1}, \cdots, n_{k}\right)$ denote the largest value of $w$ such that $p^{w}$ divides $\left(n_{1}, \cdots, n_{k}\right)$. To determine $E\left(n_{1}, \cdots, n_{k}\right)$ we shall make use of an analogue [4] of Kummer's famous theorem for binomial coefficients:

LEMMA 2.1. Let $n$ have expansion (1.1), let $n=n_{1}+\cdots+n_{k}$ and let

$$
n_{i}=a_{i, 0}+a_{i, 1} p+\cdots+2_{i, s} p^{s} \quad\left(0 \leqq a_{i, r}<p\right)
$$

for $i=1, \cdots, k . \quad$ If

$$
\begin{aligned}
a_{1,0}+\cdots+a_{k, 0} & =\varepsilon_{0} p+a_{0} \\
\varepsilon_{0}+a_{1,1}+\cdots+a_{k, 1} & =\varepsilon_{1} p+a_{1} \\
\cdots & \\
\varepsilon_{s-1}+a_{1, s}+\cdots+a_{k, s} & =a_{s}
\end{aligned}
$$

where each $\varepsilon_{i}=0,1, \cdots$, or $k-1$, then

$$
E\left(n_{1}, \cdots, n_{l}\right)=\varepsilon_{0}+\varepsilon_{1}+\cdots+\varepsilon_{s-1} .
$$

If $n$ has expansion (1.1) and if $\nu(n)$ is the largest value of $w$ such that $p^{w}$ divides $n$ !, then it is familiar $[1, p .55]$ that

$$
\nu(n)=\frac{n-S(n)}{p-1}
$$

where $S(n)=a_{0}+a_{1}+\cdots+a_{s}$. Thus we have

Lemma 2.2. If $n=n_{1}+\cdots+n_{k}$ then

$$
E\left(n_{1}, \cdots, n_{k}\right)=\frac{S\left(n_{1}\right)+\cdots+S\left(n_{k}\right)-S(n)}{p-1} .
$$

Furthermore, if $E_{t}\left(n_{1}, \cdots, n_{k}\right)$ is the largest value of $w$ such that $p^{w}$ divides

$$
(n+t) \cdots(n+1) \quad\left(n_{1}, \cdots, n_{k}\right),
$$

then

$$
E_{t}\left(n_{1}, \cdots, n_{k}\right)=\frac{S\left(n_{1}\right)+\cdots+S\left(n_{k}\right)-S(n+t)+t}{p-1} .
$$


Compositions, or ordered partitions, are important in evaluating $\theta_{j}(k ; n)$. We define a composition of a nonnegative integer $u$ into $r$ parts to be an ordered sequence of $r$ nonnegative integers whose sum is $u$. This is more general than the usual definition of composition in that we allow 0 to be one or more of the parts. See [7, pp. 124125] for example.

Throughout this paper we shall let $C(u)$ denote the number of compositions of $u$ into exactly $k$ parts, with no part larger than $p-1$. We define $C(u)=0$ if $u<0$.

LEMMA 2.3. $C(u)$ is the coefficient of $x^{u}$ in the expansion of

$$
\left(1+x+x^{2}+\cdots+x^{p-1}\right)^{k}=\left[\sum_{i=0}^{\infty}\left(\begin{array}{c}
k+i-1 \\
i
\end{array}\right) x^{i}\right]\left(1-x^{p}\right)^{k} .
$$

It is clear from Lemma 2.3 that if $0 \leqq a<p$ and if $0 \leqq b$, then

$$
C(a+b p)=\sum_{i=0}^{b}(-1)^{i}\left(\begin{array}{c}
k \\
i
\end{array}\right)\left(\begin{array}{c}
k-1+a+(b-i) p \\
k-1
\end{array}\right) .
$$

In particular, for $0 \leqq a<p$,

$$
\begin{aligned}
C(a) & =\left(\begin{array}{c}
k-1+a \\
k-1
\end{array}\right), \\
C(a+p) & =\left(\begin{array}{c}
k-1+a+p \\
k-1
\end{array}\right)-k\left(\begin{array}{c}
k-1+a \\
k-1
\end{array}\right), \\
C(a+2 p) & =\left(\begin{array}{c}
k-1+a+2 p \\
k-1
\end{array}\right)-k\left(\begin{array}{c}
k-1+a+p \\
k-1
\end{array}\right)+\left(\begin{array}{c}
k \\
2
\end{array}\right)\left(\begin{array}{c}
k-1+a \\
k-1
\end{array}\right) .
\end{aligned}
$$

3. Evaluation of $\theta_{0}(k ; n), \theta_{1}(k ; n), \theta_{2}(k ; n)$.

THEOREM 3.1. If $n$ has expansion (1.1) then

$$
\theta_{0}(k ; n)=C\left(a_{0}\right) C\left(a_{1}\right) \cdots C\left(a_{s}\right) \text {. }
$$

Proof. We use Lemma 2.1. If $E\left(n_{1}, \cdots, n_{k}\right)=0$ then we must have

$$
\sum_{i=1}^{k} a_{i, r}=a_{r} \quad(r=0, \cdots, s) .
$$

For a given $r$, the total number of ways we can have this equality is equal to $C\left(a_{r}\right)$.

Note that by Lemma 2.3 we have

$$
C\left(a_{r}\right)=\left(\begin{array}{c}
a_{r}+k-1 \\
k-1
\end{array}\right) \quad(r=0, \cdots, s) .
$$


THEOREM 3.2. If $n$ has expansion (1.1) then

$$
\theta_{1}(k ; n)=\sum_{i=0}^{s-1} C\left(a_{0}\right) \cdots C\left(a_{i-1}\right) C\left(a_{i}+p\right) C\left(a_{i+1}-1\right) C\left(a_{i+2}\right) \cdots C\left(a_{s}\right) \text {. }
$$

Proof. Using Lemma 2.1, we see that if $E\left(n_{1}, \cdots, n_{k}\right)=1$ then we must have exactly one $\varepsilon_{i}=1,0 \leqq i<s$. So for some $i$ we have

$$
\begin{aligned}
& a_{1, i}+\cdots+a_{k, i}=a_{i}+p, \\
& a_{1, i+1}+\cdots+a_{k, i+1}=a_{i+1}-1, \\
& a_{1, r}+\cdots+a_{k, r}=a_{r} \quad(r \neq i, i+1) .
\end{aligned}
$$

Clearly the total number of ways we can have these equalities is

$$
C\left(a_{0}\right) \cdots C\left(a_{i-1}\right) C\left(a_{i}+p\right) C\left(a_{i+1}-1\right) C\left(a_{i+2}\right) \cdots C\left(a_{s}\right) \text {. }
$$

To simplify the formula for $\theta_{2}(k ; n)$ we introduce the following notation. Let

$$
\begin{aligned}
A_{i} & =\left[\prod_{t=0}^{s} C\left(a_{t}\right)\right] /\left[C\left(a_{i}\right) C\left(a_{i+1}\right) C\left(a_{i+2}\right)\right], \\
B_{i} & =\left[\prod_{t=0}^{s} C\left(a_{t}\right)\right] /\left[C\left(a_{i}\right) C\left(a_{i+1}\right)\right], \\
H_{i, r} & =\left[\prod_{t=0}^{s} C\left(a_{t}\right)\right] /\left[C\left(a_{i}\right) C\left(a_{i+1}\right) C\left(a_{r}\right) C\left(a_{r+1}\right)\right] .
\end{aligned}
$$

THEOREM 3.3. If $n$ has expansion (1.1) then

$$
\begin{aligned}
\theta_{2}(k ; n)= & \sum_{i=0}^{s-2} C\left(p+a_{i}\right) C\left(p+a_{i+1}-1\right) C\left(a_{i+2}-1\right) A_{i} \\
& +\sum_{i=0}^{s-1} C\left(2 p+a_{i}\right) C\left(a_{i+1}-2\right) B_{i} \\
& +\sum_{r=i+2}^{s-1} \sum_{i=0}^{s-3} C\left(p+a_{i}\right) C\left(a_{i+1}-1\right) C\left(p+a_{r}\right) C\left(a_{r+1}-1\right) H_{i, r} .
\end{aligned}
$$

Proof. The proof is similar to the proof of Theorem 3.2. We determine the number of ways we can have exactly two of the $\varepsilon$ 's equal to 1 or exactly one $\varepsilon$ equal to 2 , and all other $\varepsilon$ 's equal to 0 .

For example, let $p=5, k=3$, and $n=278=3+5^{2}+2 \cdot 5^{3}$. We have

$$
\begin{aligned}
\theta_{0}(3 ; 278)= & C(3) C(0) C(1) C(2)=180 ; \\
\theta_{1}(3 ; 278)= & C(3) C(5) C(0) C(2)+C(3) C(0) C(6) C(1)=1650, \\
\theta_{2}(3 ; 278)= & C(8) C(4) C(0) C(2)+C(3) C(5) C(5) C(1) \\
& +C(3) C(0) C(11) C(0)=11,100 .
\end{aligned}
$$

In each example we have used (2.2) to evaluate $C(u)$.

4. Generating functions for $\theta_{\jmath}(k ; n)$. Let $\psi_{t, j}(k ; n)$ denote the 
number of products $(n+t) \cdots(n+1)\left(n_{1}, \cdots, n_{k}\right), n_{1}+\cdots+n_{k}=n$, divisible by exactly $p^{j}$. Clearly

$$
\psi_{t, j}(k ; n)=\theta_{j-r}(k ; n)
$$

if $p^{r}$ is the highest power of $p$ dividing $(n+t) \cdots(n+1)$.

Also

$$
\psi_{t, j}(k ; n)=0
$$

if $p^{j+1}$ divides $(n+t) \cdots(n+1)$. We introduce the following generating functions:

$$
\begin{aligned}
& F_{0}(x, y)=\sum_{n=0}^{\infty} \sum_{j=0}^{\infty} \theta_{j}(k ; n) x^{n} y^{j} \\
& F_{t}(x, y)=\sum_{n=0}^{\infty} \sum_{j=0}^{\infty} \psi_{t, j}(k ; n) x^{n} y^{j}
\end{aligned}
$$

Using an argument analogous to that of Carlitz [3], we obtain

$$
F_{0}(x, y)=\sum_{t=0}^{m} y^{t} f_{t}(x) F_{t}\left(x^{p}, y\right)
$$

where $m$ is the integer such that

$$
m p \leqq k(p-1)<(m+1) p
$$

and

$$
\begin{aligned}
& f_{t}(x)=\sum_{a=t p}^{t p+p-1} C(a) x^{a} \\
& (0 \leqq t<m), \\
& f_{m}(x)=\sum_{a=m p}^{k p-k} C(a) x^{a} .
\end{aligned}
$$

Comparing coefficients of $x^{n} y^{j}$ on both sides of (4.2), we have, for $0 \leqq a<p$,

$$
\theta_{j}(k ; a+b p)=C(a) \theta_{j}(k ; b)+\sum_{t=1}^{m} C(a+t p) \psi_{t, j-t}(k ; b-t) .
$$

In (4.4) it is understood that $\psi_{t, j}(k ; u)=0$ if $u<0$ and $\psi_{t,-1}(k ; u)=0$. Also, for $\mathrm{t}<p$,

$$
F_{t}(x, y)=\sum_{r=1}^{h} y^{r} g_{r}(x) F_{r}\left(x^{p}, y\right)
$$

where $h$ is the integer such that

$$
h p-t \leqq k(p-1)<(h+1) p-t,
$$


and

$$
\begin{aligned}
& g_{0}(x)=\sum_{a=0}^{p-t-1} C(a) x^{a}, \\
& g_{r}(x)=\sum_{a=r p-t}^{(r+1) p-t-1} C(a) x^{a} \quad(r=1, \cdots, h-1), \\
& g_{h}(x)=\sum_{a=h p-t}^{k p-k} C(a) x^{a} .
\end{aligned}
$$

Thus for $0 \leqq \alpha<p-t, h p+a \leqq k p-k$, we have

$$
\psi_{t, j}(k ; a+b p)=C(a) \theta_{j}(k ; b)+\sum_{r=1}^{h} C(a+r p) \psi_{r, j-r}(k ; b-r) .
$$

For $0 \leqq a<p-t, h p+a>k p-k$, we have

(4.7) $\psi_{t, j}(k ; a+b p)=C(a) \theta_{j}(k ; b)+\sum_{r=1}^{h-1} C(a+r p) \psi_{r, j-r}(k ; b-r)$.

For $p-t \leqq a<p$, we have

$$
\psi_{t, j}(k ; a+b p)=\sum_{r=1}^{h} C(a+(r-1) p) \psi_{r, j-r}(k ; a-r+1) .
$$

Here again it is understood that $\psi_{r, j}(k ; u)=0$ if $u<0$. We remark that in all of these formulas specific values for $C(u)$ can be found from formula (2.2).

Using (4.4) we can compute $\theta_{j}(k ; n)$ for special values of $n$. By (4.4) and (4.1) we have, for $0 \leqq a<p, 0 \leqq b<p$,

$$
\begin{aligned}
\theta_{j}(k ; a+b p) & =C(a+j p) \theta_{0}(k ; b-j) \\
& =C(a+j p) C(b-j) \text { if } j \leqq m, \\
& =0 \text { if } j>m
\end{aligned}
$$

where $m$ is defined by (4.3).

Also, if $0 \leqq a<p$,

$$
\begin{aligned}
\theta_{j}\left(k ; a+p^{2}\right) & =C(a) C(1) \quad \text { if } j=0, \\
& =C(a+(j-1) p) C(p-j+1) \text { if } 1 \leqq j \leqq m+1, \\
& =0 \text { if } j>m+1 .
\end{aligned}
$$

If $0 \leqq a<p, p>2$,

$$
\begin{array}{rlr}
\theta_{j}\left(k ; a+2 p^{2}\right) & \\
= & C(a+(j-2) p) \theta_{1}(k ; 2 p-j+2) & \\
& +C(a+(j-1) p) \theta_{0}(k ; 2 p-j+1) & (1<j \leqq p+1, j \leqq m+1), \\
= & C(a+(j-2) p) \theta_{1}(k ; 2 p-j+2) & (j=m+2 \leqq p+1), \\
= & C(a+(j-2) p) \theta_{1}(k ; p) & (j=p+2 \leqq m+2), \\
= & C(a+(j-2) p) \theta_{0}(k ; p-r+2) & (j=p+r \leqq m+2,2<r \leqq p+2), \\
= & 0 \text { if } j>m+2 .
\end{array}
$$


If $0 \leqq a<p, 0 \leqq b<p$,

$$
\begin{aligned}
& \theta_{j}\left(k ; a+b p+p^{2}\right) \\
& =C(a+(j-1) p) \theta_{1}(k ; p+b-j+1) \\
& +C(a+j p) \theta_{0}(k ; p+b-j) \\
& (b \geqq j ; m+1>j) \\
& =C(a+(j-1) p) \theta_{0}(k ; p+b-j+1) \quad(b<j \leqq p+b, m+1>j) \text {, } \\
& =C(a+m p) \theta_{1}(k ; p+b-m) \\
& (j=m+1, b \geqq m), \\
& =C(a+m p) \theta_{0}(k ; p+b-m) \\
& (j=m+1, b<m) \text {, } \\
& =0 \text { if } j>m+1 \text {. }
\end{aligned}
$$

Some of the results in [2] can also be generalized. We use the symbols $E\left(n_{1}, \cdots, n_{k}\right)$ and $E_{t}\left(n_{1}, \cdots, n_{k}\right)$ as they are used in Lemma 2.2 .

Let

Note that

$$
\begin{aligned}
F_{j}\left(n ; x_{1}, \cdots, x_{k}\right) & =\sum_{\substack{a_{1}+\cdots+a_{k}=n \\
E\left(a_{1}, \cdots, a_{k}\right)=j}} x_{1}^{a_{1}} \cdots x_{k}^{a_{k}}, \\
G_{t, j}\left(n ; x_{1}, \cdots, x_{k}\right) & =\sum_{\substack{a_{1}+\cdots,+a_{k}=n \\
E^{\prime}\left(a_{1}, \cdots, a_{n}\right)=j}}^{a_{1}} \cdots x_{k}^{a_{k}} \quad(t>0), \\
G_{0, j}\left(n ; x_{1}, \cdots, x_{k}\right) & =F_{j}\left(n ; x_{1}, \cdots, x_{k}\right) .
\end{aligned}
$$

$$
\begin{aligned}
F_{j}(n ; x, \cdots, x) & =x^{n} \theta_{j}(k ; n), \\
G_{t, j}(n ; x, \cdots, x) & =x^{n} \psi_{t, j}(k ; n) .
\end{aligned}
$$

By generalizing Carlitz's work in [2] in the natural way, we obtain

$$
\begin{aligned}
& F_{j}\left(a+b p ; x_{1}, \cdots, x_{k}\right) \\
& \quad=\sum_{s=0}^{m} c_{s p+a}\left(x_{1}, \cdots, x_{k}\right) G_{s, j-s}\left(b-s ; x_{1}^{p}, \cdots, x_{k}^{p}\right)
\end{aligned}
$$

where $0 \leqq a<p, m$ is defined by (4.3), and

$$
c_{r}\left(x_{1}, \cdots, x_{k}\right)=\sum_{s_{1}+\cdots+s_{k}=r} x_{1}^{s_{1}} \cdots x_{k}^{s_{k}} .
$$

Also, if $h$ is defined by (4.5),

$$
\begin{array}{r}
G_{t, j}\left(a+b p ; x_{1}, \cdots, x_{k}\right) \quad \begin{array}{r}
(h p+a \leqq k p-k, 0 \leqq a<p-t), \\
=\sum_{s=0}^{h} c_{s p+a}\left(x_{1}, \cdots, x_{k}\right) G_{s, j-s}\left(b-s ; x_{1}^{p}, \cdots, x_{k}^{p}\right) \\
=\sum_{s=0}^{h-1} c_{s p+a}\left(x_{1}, \cdots, x_{k}\right) G_{s, j-s}\left(b-s ; x_{1}^{p}, \cdots, x_{k}^{p}\right) \\
(h p+a>k p-k, 0 \leqq a<p-t), \\
=\sum_{s=1}^{h} c_{(s-1) p+a}\left(x_{1}, \cdots, x_{k}\right) G_{s, j-s}\left(a-s+1 ; x_{1}^{p}, \cdots, x_{k}^{p}\right) \\
(p-t \leqq a<p-1) .
\end{array}
\end{array}
$$


5. Some special evaluations. If $j>\nu(n)$, where $\nu(n)$ is the exponent of the highest power of $p$ that divides $n$ !, then it is clear that $\theta_{j}(k ; n)=0$. For example, if $0 \leqq a<p, 0 \leqq b<p$ then

$$
\theta_{j}(k ; a+b p)=0
$$

Let $n$ have expansion (1.1). By Lemma 2.1 it is clear that $\theta_{j}(k ; n)=0$ for $j>M$, where

$$
\begin{aligned}
M & =s(k-1) \text { if } k \leqq a_{s}+1 \\
& =(s-1)(k-1)+a_{s} \text { if } k>a_{s}+1 .
\end{aligned}
$$

Also,

$$
\begin{aligned}
& \theta_{M}(k ; n) \\
& =C\left(a_{0}+(k-1) p\right) C\left(a_{s}-k+1\right) \prod_{i=1}^{s-1} C\left(a_{i}-k+1+(k-1) p\right) \\
& \left(k \leqq a_{s}+1\right) ， \\
& =C\left(a_{0}+(k-1) p\right) C\left(a_{s-1}-k+1+a_{s} p\right) \prod_{i=1}^{s-2} C\left(a_{i}-k+1+(k-1) p\right) \\
& =C\left(a_{0}+a_{1} p\right) \\
& \left(k>a_{s}+1, s>1\right) \text {, } \\
& \left(k>a_{s}+1, s=1\right) \text {. }
\end{aligned}
$$

For example, if $k=2$ and $a_{s} \neq 0$ then $M=s$. This is the case for ordinary binomial coefficients. We have in this case

$$
\theta_{s}(2 ; n)=\left(p-a_{0}-1\right)\left(p-a_{1}\right) \cdots\left(p-a_{s-1}\right) a_{s} .
$$

For $p=2$ we can generalize the method used in [6]. Let

$$
\begin{aligned}
n & =2^{e_{1}}+\cdots+2^{e_{r}}, \quad 0 \leqq e_{1}<\cdots<e_{r}, \\
n_{i} & =2^{e_{i, 1}}+\cdots+2^{e_{i, S(i)}}, \quad 0 \leqq e_{i, 1}<\cdots<e_{i, S(i)} .
\end{aligned}
$$

Consider all the different compositions $n=n_{1}+\cdots+n_{k}$ such that (5.1) and (5.2) hold, such that

$$
S\left(n_{1}\right)+\cdots+S\left(n_{k}\right)=r+j,
$$

and such that there are a total of $r+j-t e_{i, w}$ 's having the property that $e_{i, w} \neq e_{x, y}$ for all $x, y$ (except for the one case $i=x, w=y$ ). Let $b_{j, t}$ be the sum over all these compositions of the number of different ways of distributing the remaining $t e_{i, w}$ 's into $k$ distinct cells with no two identical objects in the same cell. Then for $p=2, j>0$,

$$
\theta_{j}(k ; n)=b_{j, 2} k^{m+j-2}+b_{j, 3} k^{m+j-3}+\cdots+b_{j, m+j} .
$$

Using the convention that $e_{1}-e_{0}=t$ means $e_{1}=t-1$ and that $e_{1}-e_{0}>t$ means $e_{1}>t-1$, let 


$$
\begin{array}{rlrl}
e_{i}-e_{i-1} & >1 \text { for } q_{1} \text { terms } e_{i}, & \\
& >2 \text { for } q_{2} \text { terms } e_{i}, & \\
& =1, e_{i-1}-e_{i-2}=1 \text { for } q_{3} \text { terms } e_{i} & (i \neq 2), \\
& =1, e_{i-1}-e_{i-2}>1 \text { for } q_{4} \text { terms } e_{i}, & \\
& =2 \text { for } q_{5} \text { terms } e_{i} & & (i \neq 1), \\
& =1 \text { for } q_{6} \text { terms } e_{i} & & (i \neq 1) .
\end{array}
$$

Then, by (5.3), for $p=2$,

$$
\begin{aligned}
\theta_{0}(k ; n)=k^{r}, & \\
\theta_{1}(k ; n)= & q_{1}\left(\begin{array}{l}
k \\
2
\end{array}\right) k^{r-1}+q_{6}\left(\begin{array}{l}
k \\
3
\end{array}\right) k^{r-2}, \\
\theta_{2}(k ; n)= & q_{2}\left(\begin{array}{l}
k \\
2
\end{array}\right) k^{r}+q_{5}\left(\begin{array}{l}
k \\
3
\end{array}\right) k^{r-1} \\
& +\left[\left(\begin{array}{l}
q_{1} \\
2
\end{array}\right)+q_{4}\right]\left(\begin{array}{l}
k \\
2
\end{array}\right)^{2} k^{r-2} \\
& +\left[\begin{array}{l}
q_{4}\left(q_{1}-1\right)+q_{3} \\
3
\end{array}\right]\left(\begin{array}{l}
k \\
3
\end{array}\right)\left(\begin{array}{l}
k \\
2
\end{array}\right) k^{r-3} \\
& +\left[\left(\begin{array}{l}
q_{4} \\
2
\end{array}\right)+q_{3}\left(q_{3}-1\right)+q_{4} q_{3}\right]\left(\begin{array}{l}
k \\
3
\end{array}\right)^{2} k^{r-4} .
\end{aligned}
$$

For example, let $n=2^{4}+2^{5}+2^{20}+2^{26}+2^{28}$. Then $q_{1}=4, q_{2}=3$, $q_{3}=0, q_{4}=1, q_{5}=1$ and $q_{6}=1$. Thus

$$
\begin{aligned}
& \theta_{0}(k ; n)=k^{5} \\
& \theta_{1}(k ; n)=4\left(\begin{array}{l}
k \\
2
\end{array}\right) k^{4}+\left(\begin{array}{l}
k \\
3
\end{array}\right) k^{3}, \\
& \theta_{2}(k ; n)=3\left(\begin{array}{l}
k \\
2
\end{array}\right) k^{5}+\left(\begin{array}{l}
k \\
3
\end{array}\right) k^{4}+7\left(\begin{array}{l}
k \\
2
\end{array}\right)^{2} k^{3}+3\left(\begin{array}{l}
k \\
3
\end{array}\right)\left(\begin{array}{l}
k \\
2
\end{array}\right) k^{2} .
\end{aligned}
$$

\section{REFERENCES}

1. P. Bachmann, Niedere Zahlentheorie, vol. 1, Leipzig, 1902.

2. L. Carlitz, Distribution of binomial coefficients, Riv. Mat. Univ. Parma, (2) 11 (1970), 45-64.

3. - The number of binomial coefficients divisible by a fixed power of a prime, Rend. Circ. Mat. Palermo, (2) 16 (1967), 299-320.

4. Robert D. Fray, Congruence properties of ordinary and q-binomial coefficients, Duke Math. J., 34 (1967), 467-480.

5. F. T. Howard, Formulas for the number of binomial coefficients divisible by a fixed power of a prime, Proc. Amer. Math. Soc., 37 (1973), 358-362. 
6. F. T. Howard, The number of binomial coefficients divisible by a fixed power of 2, Proc. Amer. Math. Soc., 29 (1971), 236-242.

7. J. Riordan, An Introduction to Combinatorial Analysis, New York, 1958.

Received September 11, 1972.

WAKEFFOREST UNIVERSITY 


\section{PACIFIC JOURNAL OF MATHEMATICS}

\section{EDITORS}

RICHARD ARENS (Managing Editor) University of California

Los Angeles, California 90024

R. A. BeaUmont

University of Washington Seattle, Washington 98105
J. DUGUNDJI*

Department of Mathematics University of Southern California Los Angeles, California 90007

D. Gilbarg and J. Milgram Stanford University

Stanford, California 94305

\section{ASSOCIATE EDITORS}

E. F. BECKENBACH

B. H. NeUmanN

F. WOLF

K. YoSHIDA

\section{SUPPORTING INSTITUTIONS}

\section{UNIVERSITY OF BRITISH COLUMBIA CALIFORNIA INSTITUTE OF TECHNOLOGY UNIVERSITY OF CA.LIFORNIA MONTANA STATE UNIVERSITY UNIVERSITY OF NEVADA NEW MEXICO STATE UNIVERSITY OREGON STATE UNIVERSITY UNIVERSITY OF OREGON OSAKA UNIVERSITY}

UNIVERSITY OF SOUTHERN CALIFORNIA STANFORD UNIVERSITY UNIVERSITY OF TOKYO UNIVERSITY OF UTAH WASHINGTON STATE UNIVERSITY UNIVERSITY OF WASHINGTON AMERICAN MATHEMATICAL SOCIETY NAVAL WEAPONS CENTER

* C. R. DePrima California Institute of Technology, Pasadena, CA 91109, will replace J. Dugundji until August 1974. 


\section{Pacific Journal of Mathematics}

\section{Vol. 50, No. $1 \quad$ September, 1974}

Gail Atneosen, Sierpinski curves in finite 2-complexes.............. 1

Bruce Alan Barnes, Representations of $B^{*}$-algebras on Banach spaces .... 7

George Benke, On the hypergroup structure of central $\Lambda(p)$ sets ....... 19

Carlos R. Borges, Absolute extensor spaces: a correction and an

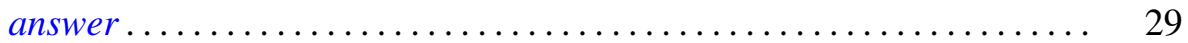

Tim G. Brook, Local limits and tripleability .................. 31

Philip Throop Church and James Timourian, Real analytic open maps .... 37

Timothy V. Fossum, The center of a simple algebra ............... 43

Richard Freiman, Homeomorphisms of long circles without periodic

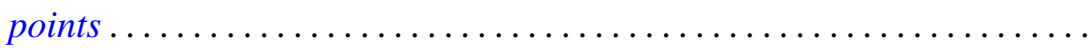

B. E. Fullbright, Intersectional properties of certain families of compact

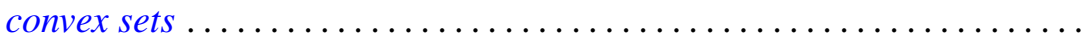

Harvey Charles Greenwald, Lipschitz spaces on the surface of the unit

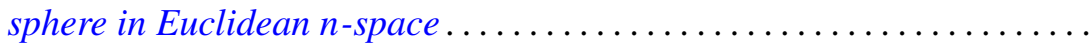

Herbert Paul Halpern, Open projections and Borel structures for

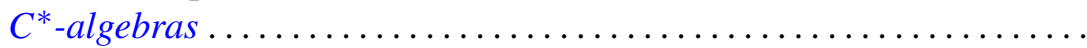

Frederic Timothy Howard, The numer of multinomial coefficients divisible

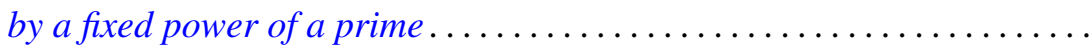

Lawrence Stanislaus Husch, Jr. and Ping-Fun Lam, Homeomorphisms of manifolds with zero-dimensional sets of nonwandering points........ 109

Joseph Edmund Kist, Two characterizations of commutative Baer rings ...

Lynn McLinden, An extension of Fenchel's duality theorem to saddle functions and dual minimax problems ................

Leo Sario and Cecilia Wang, Counterexamples in the biharmonic classification of Riemannian 2-manifolds...

Saharon Shelah, The Hanf number of omitting complete types ...

Richard Staum, The algebra of bounded continuous functions into a

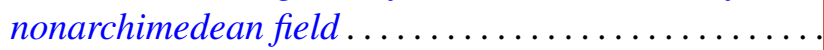

James DeWitt Stein, Some aspects of automatic continuity ..

Tommy Kay Teague, On the Engel margin

John Griggs Thompson, Nonsolvable finite groups all of whose local subgroups are solvable, $V \ldots \ldots \ldots \ldots \ldots \ldots \ldots \ldots$

Kung-Wei Yang, Isomorphisms of group extensions 